\title{
Active microfluidic transport in two-dimensional handlebodies $^{\dagger}$
}

\begin{abstract}
Jérôme Hardoüin ${ }^{1,2}$, Justine Laurent ${ }^{3,4}$, Teresa Lopez-Leon ${ }^{3,4}$, J. Ignés-Mullol ${ }^{1,2}$ and Francesc Sagués ${ }^{1,2}$

Unlike traditional nematic liquid crystals, which adopt ordered equilibrium configurations compatible with the topological constraints imposed by the boundaries, active nematics are intrinsically disordered because of their self- sustained internal flows. Controlling the flow patterns of active nematics remains a limiting step towards their use as functional materials. Here we show that confining a tubulin-kinesin active nematic to a network of connected annular microfluidic channels enables controlled directional flows and autonomous transport. In single annular channels, for narrow widths, the typically chaotic streams transform into well-defined circulating flows, whose direction or handedness can be controlled by introducing asymmetric corrugations on the channel walls. The dynamics is altered when two or three annular channels are interconnected. These more complex topologies lead to scenarios of synchronization, anti-correlation, and frustration of the active flows, and to the stabilisation of high topological singularities in both the flow field and the orientational field of the material. Controlling textures and flows in these microfluidic platforms opens unexplored perspectives towards their application in biotechnology and material science.
\end{abstract}

\section{Introduction}

Flowing active matter provides a fascinating arena to investigate a variety of phenomena in non-equilibrium Physics $\frac{12}{12}$. Potential applications include drug delivery, energy harvesting, metamaterial design, microreactor engineering, or memory storage ${ }^{3+6}$. The challenge is to make active materials functional by programming them to perform pre-defined tasks, for instance through the engineering of devices able to induce well-defined flow networks in the material. In this line, recent works have established conceptual foundations for developing autonomous microfluidic transport devices driven by flowing active matter ${ }^{7}$.

In-vitro preparations, in particular those based on the cooperative action of microtubules and protein-based motors ${ }^{8}$, hold big promises in this exciting venture. Under non-equilibrium conditions, secured by adenosine-triphosphate (ATP) feeding, these components self-organize into aqueous-based active gels ${ }^{9}$. Their two-dimensional realizations, which have received special attention in the last years, bear strong resemblance with internally

\footnotetext{
${ }^{1}$ Departament de Ciència de Materials i Química Física, Universitat de Barcelona, Martí i Franquès 1, 08028, Barcelona, Spain

${ }^{2}$ Institute of Nanoscience and Nanotechnology (IN2UB), Universitat de Barcelona, Barcelona, Spain

${ }^{3}$ Laboratoire de Physique et Mécanique des Milieux hétérogènes (PMMH), CNRS, ESPCI Paris, PSL Research University, Paris, France

${ }^{4}$ Laboratoire Gulliver, UMR CNRS 7083, ESPCI Paris, PSL Research University, Paris, France

$\dagger$ Electronic Supplementary Information (ESI) available: supplementary figures and videos. See DOI: 10.1039/cXsm00000x/
}

sheared colloidal liquid crystals 10 . They are referred to as active nematics and appear as dense, defect-laden, arrangements of bundled microtubules permanently subjected to mixing flows, that are apparently deprived of any large-scale coherence $11-16$. While considerable theoretical effort has been dedicated to describe these active nematics ${ }^{17-25}$, strategies pursuing the control of their chaotic flows have just started to be developed $14 \mid 26$.

Recent studies have shown the adaptability of biological fluids to confinement, from bacterial baths ${ }^{27+\sqrt[31]{3}}$, to cell tissues $32,-35$ or cytoplasmatic preparations ${ }^{36}$. In particular, microtubule-based systems have demonstrated to respond to frictional/viscous gradients ${ }^{1426}$ or to geometric and topological constrains by organizing their chaotic motion into regular spatio-temporal flow patterns 37,44 . Theoretical and numerical studies have also assessed the impact of confinement in active motility $45+58$, predicting a rich variety of behavior emerging from the interplay between geometry of the confining space, intrinsic order of the active material and collective flows.

In this work, we explore this interplay by confining a microtubule-based active nematic into microdevices where the active flows are organized by the geometry and topology of connected annular channels (handlebodies). We demonstrate that this type of confinement generates surprisingly coherent flow patterns. Below a critical confinement width, a regime of autonomous directed transport with random handedness emerges in isolated annuli. This flow symmetry-breaking is accompanied by a polarization of the nematic order in the direction of the 
flow. Adding asymmetric corrugations to the boundaries enables to control the transport handedness in the annulus. In the case of two interconnected annuli, we show the possibility to generate stagnation points in the active flow pattern, thereby localizing points of high topological charge in space. Finally, we uncover regimes of dynamic flow synchronization, anti-correlation and frustration when considering higher order topologies of connected annuli. These results witness the rich interplay between confinement, order and activity in active nematics, thereby extending the emerging field of topological microfluidics so far restricted to passive liquid crystals 59 . Our results also demonstrate the potential of these synthetic active materials, which can extend over large interfaces and fill complex microfluidic circuits, to program pre-designed flow patterns that could be exploited for applications in biotechnology or material science, such as cargo transport, metamaterial design, soft robotics, or life-on-a-chip systems.

\section{Results and discussions}

\subsection{Tubulin-kinesin active nematic confined to annular channels}

The active system used in this study is composed of microtubules powered by two headed kinesin molecular motor clusters 11 . Addition of the depleting agent polyethylene glycol (PEG) concentrates the microtubules into bundles, hundreds of microns long. Within each bundle, the kinesin motors bridge neighboring microtubules and walk towards their positive ends, leading to internal sliding of filaments of opposite polarity. The active nematic layer is prepared using an open-cell design 44/60, in which a microdroplet of the active aqueous microtubule-mixture is placed inside a custom-made pool of $5 \mathrm{~mm}$ diameter and subsequently covered with 100 cSt silicon oil (see Supplementary Information). Within 30 minutes, an active nematic layer a few microns thick ${ }^{61}$ extends over the whole surface of the pool, at the interface between the oil and the aqueous phase.

Our micro-devices are placed right at the interface where the active nematic is formed. They are flat, rigid polymeric slabs $100 \mu \mathrm{m}$ thick, obtained through micro-printing, in which the micro-channels are embedded. These micro-channels are holes in the polymeric matrix where the active nematic can condensate, inducing lateral confinement in the two-dimensional nematic system 44 (see Experimental Section). In contrast with traditional microfluidic devices obtained by micro-fabrication, such as softlithography, our devices do not contain inlets or outlets for the fluids, since the flows are self-sustained by the activity of the material. Because they are produced by micro-printing, the number, shape and topology of the embedded channels can be tuned at will. The geometries/topologies tested in this work belong to the class of $2 D$ handle-bodies. They are composed of connected elementary annuli. We will refer to them by using their genus number, $g$, which indicates the number of holes in the device: $g=1$ indicates a single annulus, $g=2$, two connected annuli, and so on, as shown in Fig. 7(b). In this work, we have studied the dynamics of active nematics confined to handle-bodies up to $g=3$.

\subsection{Transport and polarization in isolated annuli}

Under lateral confinement, active nematic flows reorganize into regular patterns 44 , where the spatial distribution, size, and velocity of topological defects dramatically depend on the confining parameters. While, for straight channels, the control parameter is the channel width, annular confinement adds another control parameter: the mean curvature of the channel. In order to decouple the effects of these two parameters, the inner radius is kept constant at $R_{i}=150 \mu \mathrm{m}$ and the outer radius is varied so that the annulus width spans a range of $40<w<300 \mu \mathrm{m}$.

For narrow annuli, typically below $80 \mu \mathrm{m}$, and for the material parameters used here, we observe the emergence of a continuous directed flow, which is not possible in straight channels because of boundary effects at the ends. Its handedness is random, but persistent over the whole duration of the experiment, until activity dies out. In our experiments, the velocity field is obtained by applying Particle Image Velocimetry (PIV) algorithms to the confocal fluorescence images, using the evolving features of the active nematic as flow tracers (Fig. S1(a)). The velocity field is resolved in space and time and projected onto the radial and azimuthal directions: $v_{r}(r, \theta, t)$ and $v_{\theta}(r, \theta, t)$ respectively. The time-averaged azimuthal velocity, $\bar{v}_{\theta}$, is uniform along the annulus circumference as shown in Fig. 1 (a). The color map overlaid on each fluorescence image shows values of $v_{\theta}$ above $0.2 \mu \mathrm{m} / \mathrm{s}$, so regions with negligible velocity remain colorless for a better contrast. $\bar{v}_{\theta}$ only depends on the radial position across the channel width, with an apparent slow-down towards the walls. We call this regime the transport state.

For wider annuli, typically $80<w<200 \mu \mathrm{m}$, the flow pattern is less stable in time [Fig. 1](b)]. Transport may be observed for a while, before breaking down into a chaotic regime. Occasionally the symmetry breaks again, such that transport and chaos never cease to compete over time. The transition between both states occurs randomly, each of them persisting from a few seconds to minutes before breaking down. In this particular state, that we will refer to as the switching state, the transport chirality changes randomly between two turbulent phases.

Above $200 \mu \mathrm{m}$, the flows are essentially chaotic rendering turbulent-like currents. As shown in the overlaid color map in Fig. 1.(c), $\bar{v}_{\theta}$ is less than $0.2 \mu \mathrm{m} \mathrm{s}^{-1}$ everywhere around the annulus. Experimental videos of the transport (Movie $S 1$ ), switching (Movie S2) and turbulent (Movie S3) states are available in the Supplementary Information.

In order to assess the role of the mean curvature of the annular channels, we have compared the flow profiles of concentric channels whose inner radii span the range $150-800 \mu \mathrm{m}$ and width $60 \mu \mathrm{m}$ (Fig. S2). All the channels exhibit the transport state, with velocity profiles that are equivalent, within error bars, indicating that the mean channel curvature plays a subdominant role in the system.

Given the observed rotational invariance of the velocity in the transport state, we eliminate the azimuthal dependence by computing the average tangential velocity profile $u_{t}(\tilde{r})=\left\langle\bar{v}_{\theta}\right\rangle_{\theta}(\tilde{r}$ denotes the scaled radial coordinate). The resulting data yields a symmetric convex profile, with maximum velocity at the center of 

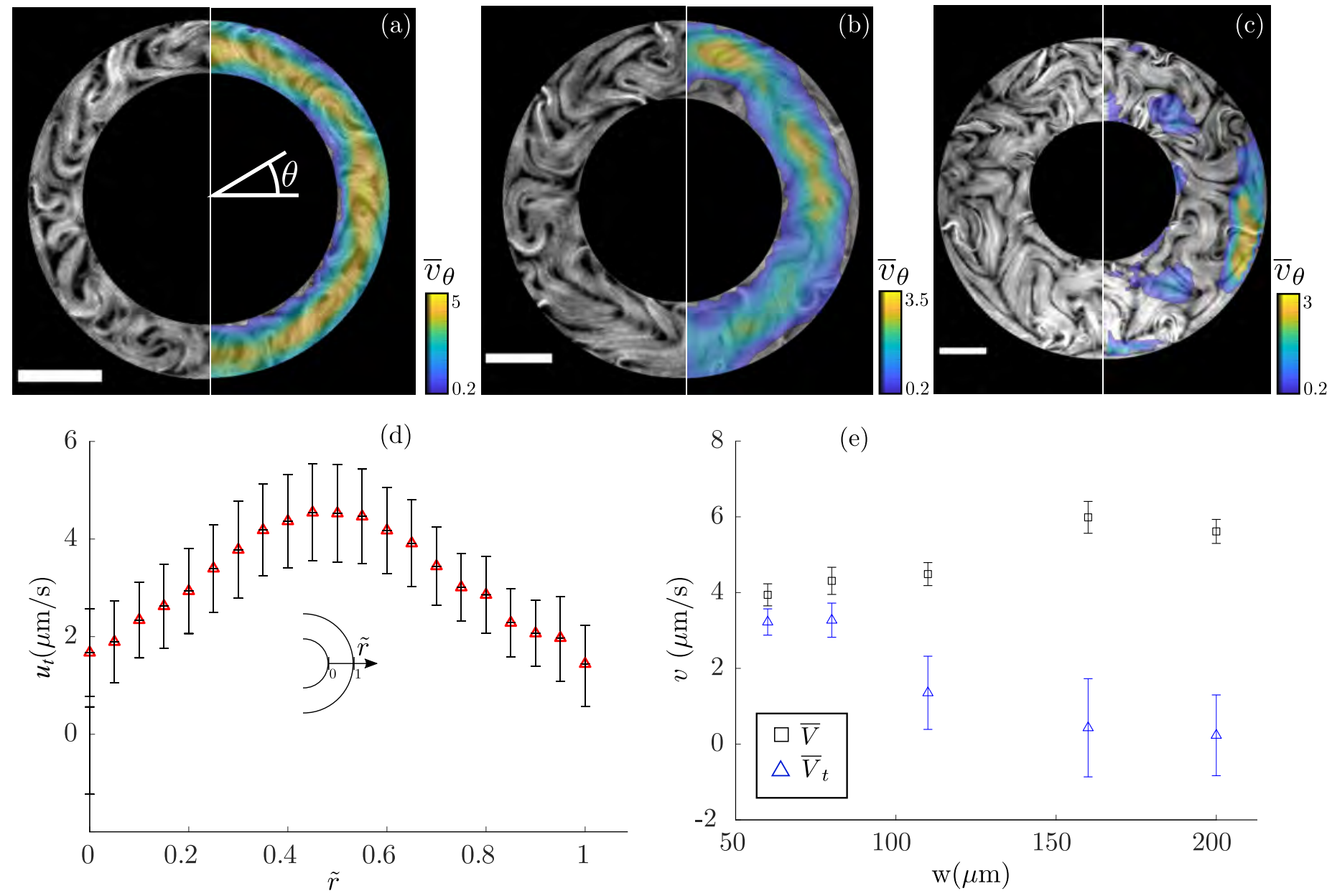

Fig. 1 Active flows confined in annuli. Panels a-c are composed of a fluorescence micrograph of the confined active nematic on the left half, and the intensity map of the time-averaged azimuthal component of the velocity on the right half. Images are overlapped with a black mask around the region of interest, for a better visualization. The lower limit of the colormap is set to $0.2 \mu \mathrm{m} \mathrm{s}^{-1}$ such that regions with negligible velocity remain colorless for a better contrast (specially in panel (c)). The annuli widths are (a) $60 \mu \mathrm{m}$ (b) $160 \mu \mathrm{m}$, and (c) $200 \mu \mathrm{m}$. Scale bars: $100 \mu \mathrm{m}$. (d) Time-averaged profile of the velocity tangential to the walls, $u_{t}(\tilde{r})=\left\langle\bar{v}_{\theta}\right\rangle_{\theta}$, as a function of the normalized radial coordinate $\tilde{r}=\left(r-R_{i}\right) /\left(R_{o}-R_{i}\right)$, where $R_{i}$ and $R_{o}$ denote the annulus inner and outer radius, respectively. (e) Mean global speed, $\bar{V}$, and azimuthal velocity, $\bar{V}_{t}$, as a function of annulus width (see text for corresponding definitions). Error bars correspond to the standard deviation of the measurements with time.

the annulus cross-section (see Fig. 1 (d)). Previous simulations 58 have predicted the emergence of a Poiseuille profile. A parabolic fit to the experimental data is proposed in Fig. S1(b). The equation of the fit has the form $u_{t}=u_{0} \cdot \tilde{r}(1-\tilde{r})$ with $u_{0}=18 \mu \mathrm{m} . \mathrm{s}^{-1}$. The agreement with the experimental flow profile is satisfactory around the center of the cross-section. However, the Poiseuille profile seriously underestimates the velocity at the walls (slip velocity). While simulations imposed a no-slip boundary condition 58 , in our experiment there seems to be low friction at the walls, as evidenced by the linear shear flow profile observed in earlier experiments with narrow rectangular channels 44 .

To quantify how much of the active flow is devoted to transport, we compute two space independent observables, $V(t)=$ $\langle v(r, \theta, t)\rangle_{\Omega}$ and $V_{t}(t)=\left\langle v_{\theta}(r, \theta, t)\right\rangle_{\Omega}$, which are the mean total velocity and the mean azimuthal velocity, respectively, averaged in space. The contribution to transport is given by the ratio $q(t)=V_{t} / V$. For a series of experiments [Fig. S1(c)], this parameter fluctuates around 0.8 in the transport regime, around
0.3 with large fluctuations in the switching state, and becomes noisy with vanishing transport in the turbulent state. By plotting the time-averaged velocities $\bar{V}_{t}$, the decay of active transport with the channel width becomes clear (Fig. 1(e)) despite an apparent increase of the mean speed $\bar{V}$.

The observed symmetry breaking can be more readily quantified in the form a scalar flow-order parameter, denoted $\hat{S}$, whose values are bounded between -1 and +1 . The value of $\hat{S}$ is obtained from kymographs of intensity profiles along a circumference at the center of the annulus, as explained in the Methods section and in Fig. 2 (a-f), providing an alternative description of the transport regime. According to our definition, $\hat{S}=+1$ (resp. $\hat{S}=-1$ ) denotes an ideal clockwise CW (resp. counter-clockwise CCW) transport current, while values close to zero correspond to the turbulent state. Typical evolutions of $\hat{S}(t)$ for different channel widths are displayed in Fig. 2(g). In the transport state, $\hat{S}$ remains very close to 1 with little fluctuations. On the contrary, $\hat{S}$ fluctuates dramatically in the switching and turbulent states, 

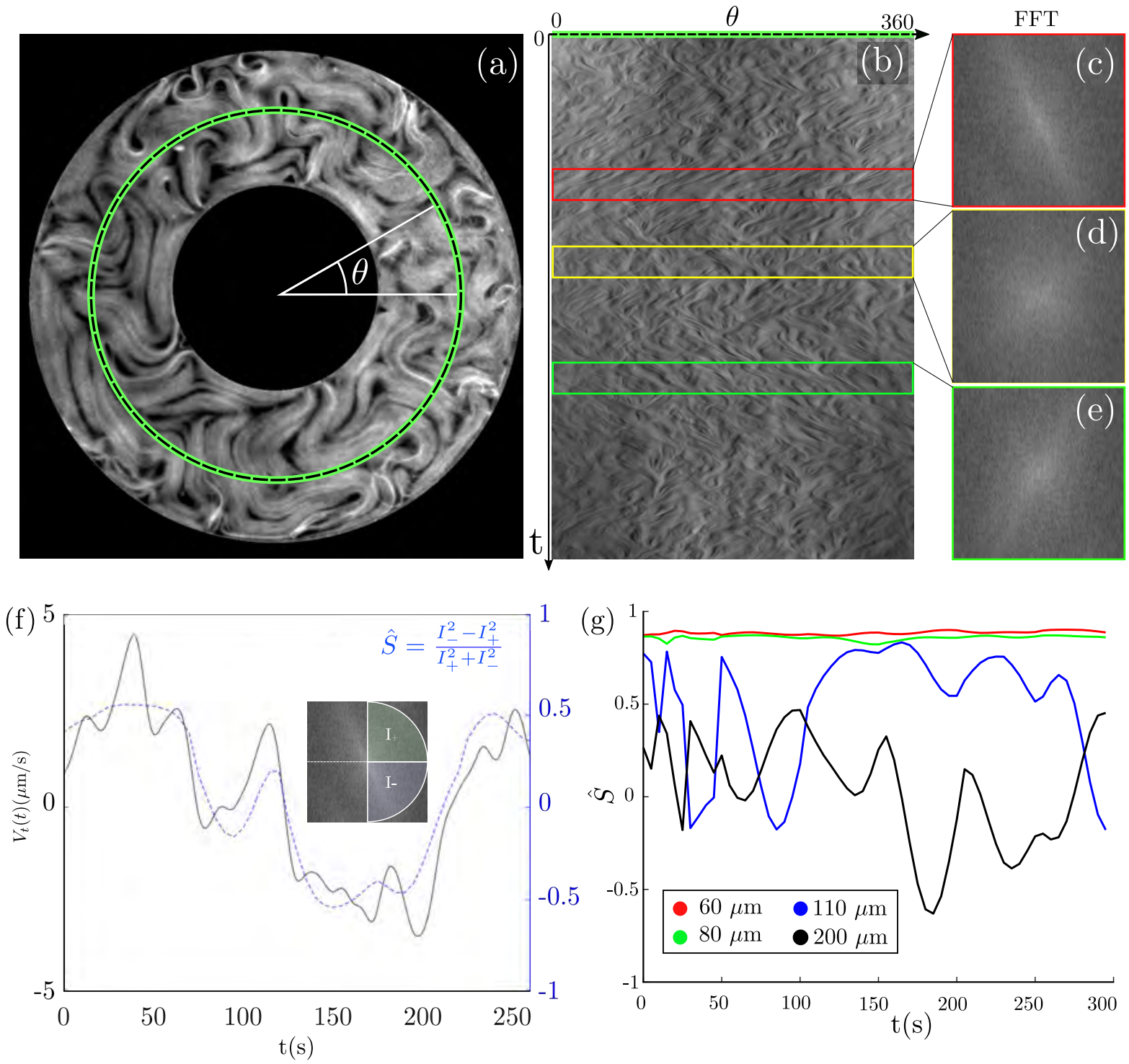

Fig. 2 Flow order in the switching state. (a) Fluorescence micrograph of an active nematic confined in a $160 \mu$ m-wide annulus. The pixel line used to construct the kymographs of the annulus dynamics is overlaid in green. The image is overlapped with a black mask around the annulus, for a better visualization.(b) Kymograph of the fluorescence intensity profile along the ring in (a). (c) Typical FFT image during clockwise transport. The stripes with a negative slope in the time series result in a bright band with a negative slope in the FFT. (d) Typical FFT image during the turbulent phase. The intensity is uniform in all directions. (e) Typical FFT image during counter-clockwise transport. (f) Flow order parameter $\hat{S}$ as a function of time compared with the PIV measurement of the azimuthal velocity for a $160 \mu \mathrm{m}$ wide annulus in the switching state. $\hat{S}$ compares the integrated intensity of the FFT image between the top-right and bottom right quadrants. $\hat{S}=+1$ (resp. $\hat{S}=-1$ ) corresponds to clockwise CW (resp. counter-clockwise CCW) flows (see Experimental Section). (g) Time evolution of $\hat{S}$, for different channel widths. 

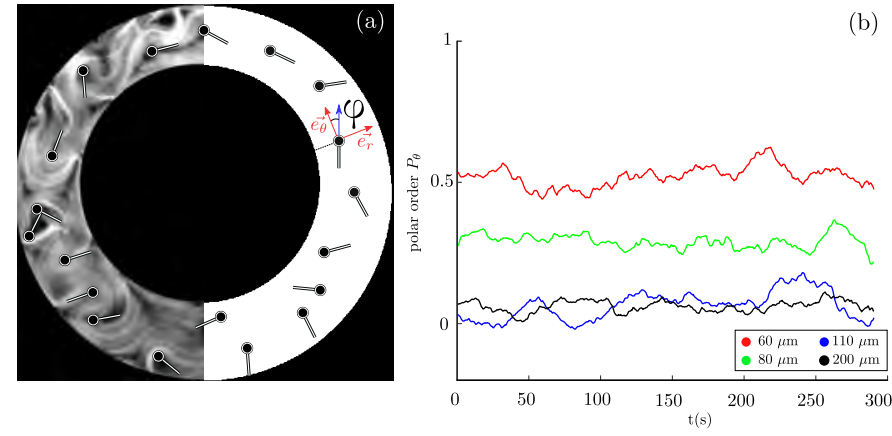

Fig. 3 Defect polarization in the active nematic annular flow. Positive defects positions and orientations are detected as described in the Methods section. Defect orientations are then projected onto the local azimuthal direction (angle $\varphi$ in (a)). (a) Fluorescence micrograph of an active nematic confined in a $80 \mu \mathrm{m}$ annulus. The image is overlapped with a black mask around the region of interest, for a better visualization. Positive defects positions and orientations are overlaid. (b) Azimuthal polar order of positive defects, $P_{\theta}=\langle\cos \varphi>$ as a function of time, for four different annuli width. See Methods for details on the computation of $P_{\theta}$.

with frequent drops towards $\hat{S}=0$, characteristic of an absence of transport. Typically, events of transport are associated to values of $|\hat{S}|>0.5$.

As usually observed in active nematics, flows are largely correlated with the organization of positive defects 10 (Fig. 3). For all but the widest annuli, positive defects acquire a remarkable polar order, denoted $P_{\theta}(t)$, measured along the azimuthal direction $\left(P_{\theta}(t)=<\cos \varphi(t)>\right.$ where $<.>$ denotes the average over the defect population a time $t$, see Methods), as shown in Fig. 3 (a). $P_{\theta}(t)$ fluctuates in time about a well-defined mean value, which is largest for the narrowest annuli (Fig. 3 (b)). The distribution of orientations, displayed in Fig. S3 (a) gives a clear signature of polar order, with a high peak in the azimuthal direction $(\varphi=0)$. Interestingly, we also notice two minor peaks along the radial direction, that correspond to the radial approaching of positive defects towards the wall, a mechanism that seems to protect defects from annihilation.

The directional interactions between positive defects and walls described above open new possibilities to control active flows through "smart" geometrical confinement, where the geometry of the micro-device would be pre-designed to induce a well defined flow pattern. For instance, in the transport state, a simple way to break the chiral symmetry without altering the topology of the channels consists in using square tracks and imposing a symmetric arrangement of indentations at either side of each of the four corners (see Fig. S4). This minor intervention suffices to control the chirality of the flow, either CW (indentation located to the right of the corner) or CCW (indentation located to the left of the corner).

\subsection{Cross-talks between flows and order in connected annuli}

In the previous section, we studied the evolution of active flows confined to elementary annuli, i.e. $g=1$ handlebodies. We now explore the effect of more complex topologies, first by studying $g=2$ handlebodies, where two annular channels are connected with increasing degrees of overlapping (see Fig. 4). The width of each annular channel is fixed at $w=80 \mu \mathrm{m}$, at the transition between transport and switching states. This dynamically rich flow regime enables to observe that the normally random and uncorrelated switching events in each annulus become synchronized, and that this process is mediated by the formation of a stable defect structure in the overlapping region.

For the smallest overlap (Fig. 4 (a)), flows and defect landscapes behave very similarly to isolated annular channels. The flow order parameter (Fig. 4 (b)) reveals that annulus number 2 (red line) has a persistent $\mathrm{CW}$ transport, while annulus number 1 (blue line) is in the switching state. This is a clear evidence that active flows are not synchronised. The flow patterns (Fig. 4 (c)) do not give any sign of interpenetration between the two annuli. Finally, the defect distribution shows an accumulation of negative defects close to the walls and a higher concentration of positive defects at the center (Fig. 4(d)), which is a sign of the confinement-induced defect unbinding reported for the case of rectangular channels 44 .

On the contrary, in the case of the largest overlap (Fig. 4 (e)), the values of the flow order parameter in both annular channels (Fig. 4 (f)) are perfectly anti-correlated. This tendency to rotate with opposite chirality is necessary to preserve the continuity of the velocity field in the overlapping region, and has also been reported in interacting bacterial vortices ${ }^{30}$. This anti-correlation is persistent within episodic switching bursts in which the order parameter $\hat{S}$ changes in a symmetric and synchronized fashion in the two coupled annuli. The corresponding flow pattern (Fig. 4 (g)) features an interesting top-down symmetry breaking. The bright yellow spot located below the cusp connecting the annuli is the signature of a strong jet of active material from that cusp towards the center. On both sides of the cusp one can also notice two blue spots that are the signature of stagnation points in the flow field. These stagnation points are attributed to recirculation vortices on each side of the jet.

The case of intermediate overlap (Fig. 4 (i)) suggests the existence of an intricate coupling between defects and flow patterns. The flow order parameter (Fig. 4 (j)) gives evidence of anti-correlations between the flow regimes in the two annuli. However, one can also observe that the average value of $\hat{S}$, below 0.5 , is significantly lower than either in less overlapping or more overlapping annular channels. As a matter of fact, the dynamics in this configuration is mostly turbulent, with no sign of transport. In spite of this, the flow pattern (Fig. $4(\mathrm{k})$ ) appears strikingly regular, with 5 equidistant stagnation points centered in the intersection region and 2 jets located at either cusp of the $g=2$ handlebody. Similarly, defect distributions are also very well organised (Fig. 4(1)). Negative defects concentrate at two locations along the main axis of the $g=2$ handlebody (blue dotted circles). On the other hand, positive defects concentrate in the vicinity of the top and bottom cusps (green dotted circles). Remarkably, there is an apparent topological protection of the center of symmetry of the $g=2$ handlebody, which is defect-free at all times. Instead, this region features a thick active bundle oriented parallel to the main axis of the $g=2$ handlebody. Apparently, jets coming from the facing cusps align the active 

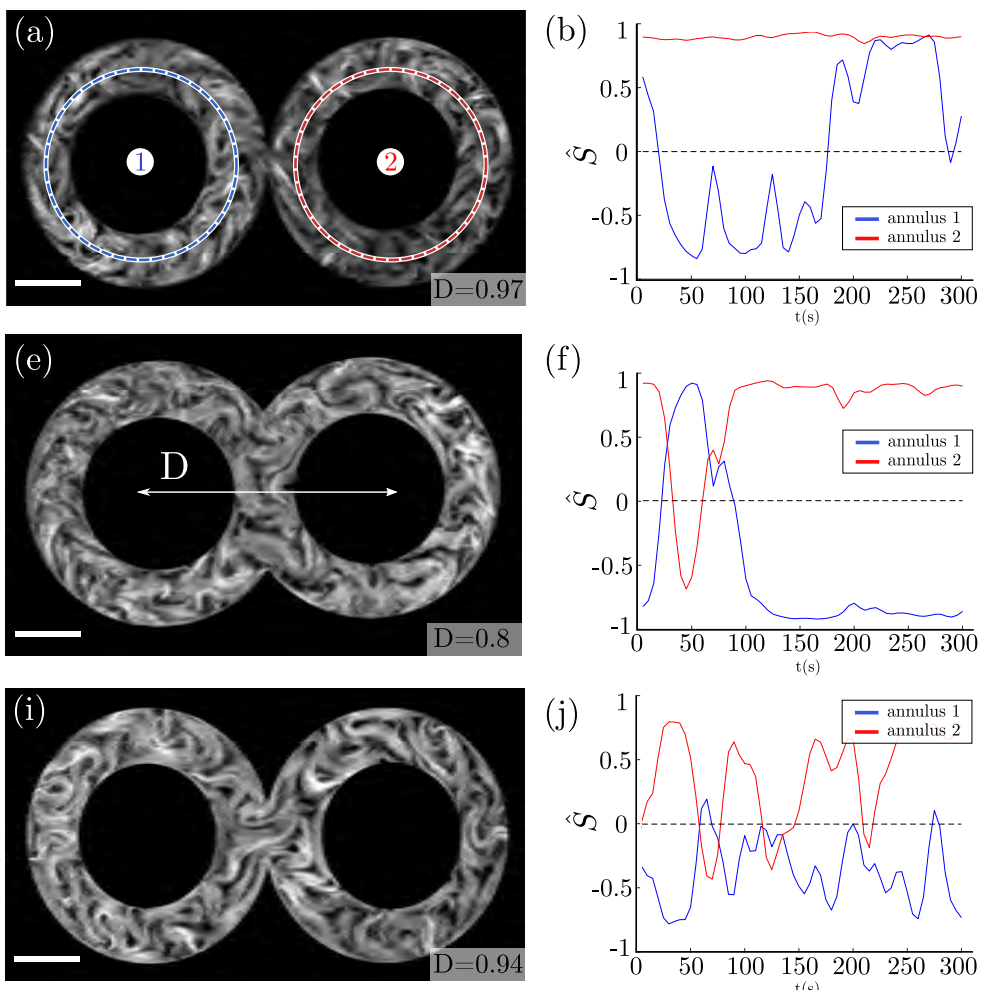

(f)
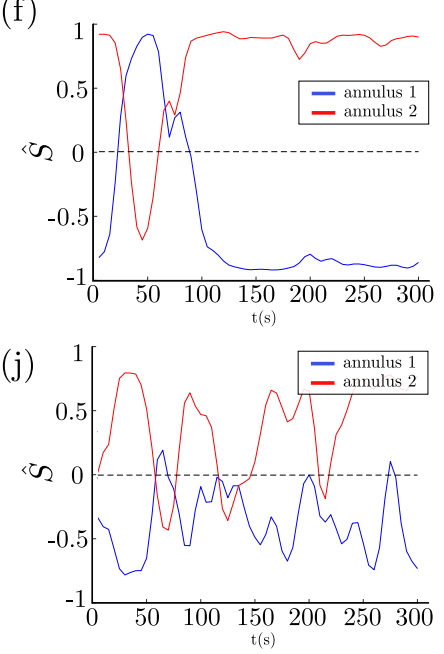

(c)

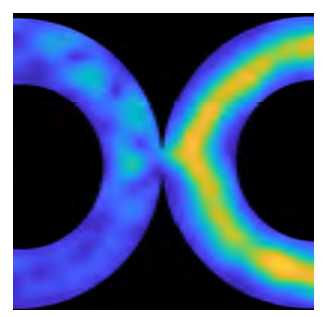

(g)
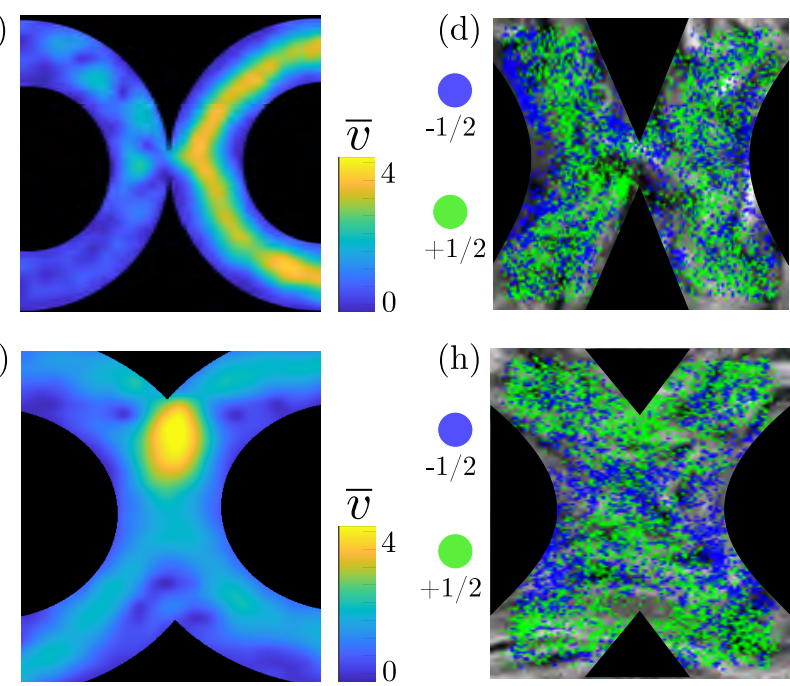

(k)

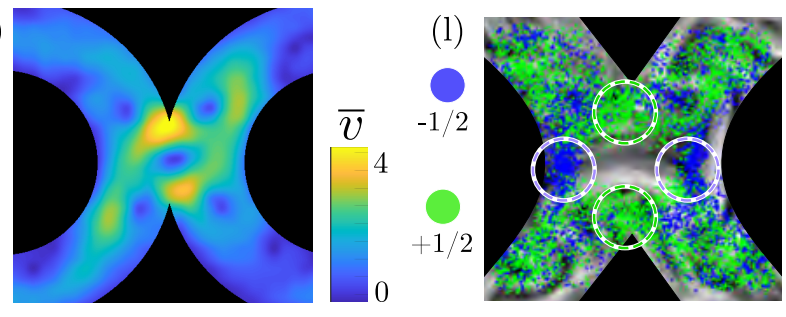

Fig. 4 Active flows in annuli connected in a genus two handlebody design. (a, e, i) Fluorescence micrographs of $80 \mu \mathrm{m}$ wide annuli connected side by side, for various inter-center distance D. (a) $D / 2 R_{o}=0.97$ (e) $D / 2 R_{o}=0.8$. Images are overlapped with a black mask around the region of interest, for a better visualization. (i) $D / 2 R_{o}=0.94(\mathrm{~b}, \mathrm{f}, \mathrm{j})$ Flow order parameter as a function of time for each annulus in (a, e, i) connected annuli. Scale bars: $100 \mu \mathrm{m}$. (c, g, k) Local speed averaged over time at the center of (a, e, i). (d, h, l) Overlay of positive (green) and negative (blue) defects positions at the center of $(\mathrm{a}, \mathrm{e}, \mathrm{i})$ connected annuli. The overlay comprises detections over 600 frames. 
material in this area, rendering defect crossing rather unlikely. The corresponding dynamics are observable in Movie S4. This scenario points to the existence of a cross-talk between singularities in co-evolving fields, velocity and nematic orientation in this case. This principle was recently raised by Giomi et al. in a study of passive liquid crystals forced through microfluidic channels ${ }^{62}$, where the geometry of the channels led to the stabilisation of topological defects that were not energetically favoured. The scenario presented here is an active analogue, where the flows are not directly prescribed, but rather emerging from the coupling between the activity of the anisotropic material and the geometry and topology of the microfluidic device.

The above flow patterns can be reconstructed from the superposition of the ideal flow fields around individual defects, as derived by Giomi et al. $[3$. From the outcome of defect tracking displayed in Fig. 4 (1), we have computed an average local topological charge, $T=1 / 2\left(N_{\text {pos }}-N_{\text {neg }}\right) / N_{\mathrm{f}}$, where $N_{\text {pos }}$ (resp. $\left.N_{\text {neg }}\right)$ is the total number of positive (resp. negative) defects detected at a given location for a period of time, and $N_{f}$ is the total number of analyzed images. The resulting topological landscape (Fig. 5 (a)) confirms the charge localization seen in the experiments and corresponding velocity maps (Fig. 5(b)). In the model, the spots of positive (resp. negative) charges are chosen as flow "sources", that generate the theoretical flow pattern of a positive (resp. negative) defect (Fig. 5 (c)). Although this "toy model" neglects interaction between flow sources, it is useful as a means to understand the interplay between flow and orientational fields. The acceleration areas in the cusp are efficiently recovered, as well as the stagnation points, although the latter appear more spread out. Note that in the representation of the computed flow fields (Fig. 5 (d)), the overlaying mask of the $g=2$ handlebody is only illustrative, and no boundary conditions are imposed in the calculations.

\subsection{Frustated topology with a genus 3 topology}

In this section, we investigate how the active nematic flows respond to geometric frustration. In the case of the $g=2$ handlebody, we have seen that the degrees of freedom in the flow are already more constrained than in the case of isolated annuli: the transport chiralities in both annular channels are necessarily antisynchronized in order to minimise the friction forces within the connecting area, as shown in Fig. 4 (e). Despite a decrease in the degrees of freedom, transport was topologically allowed in all annuli. This is no longer true in the case of the $g=3$ handlebody geometry shown in Fig. 6 (a). In this geometry, the third annular channel is connected to the other two. To get antisynchronized with both of them, the circulation inside the third channel needs to be CW and CWW at the same time, which is not possible, leading to frustration. The transport dynamics in each annular channel, presented in Fig. 6 (b), shows strong evidence of frustration. At time $t=0$, annuli number 2 (red line) and 3 (green line) are anti-correlated, with the first one rotating CW and the second one CCW. On the other hand, annulus number 1 (blue line) is in a turbulent regime. This state is not stable in
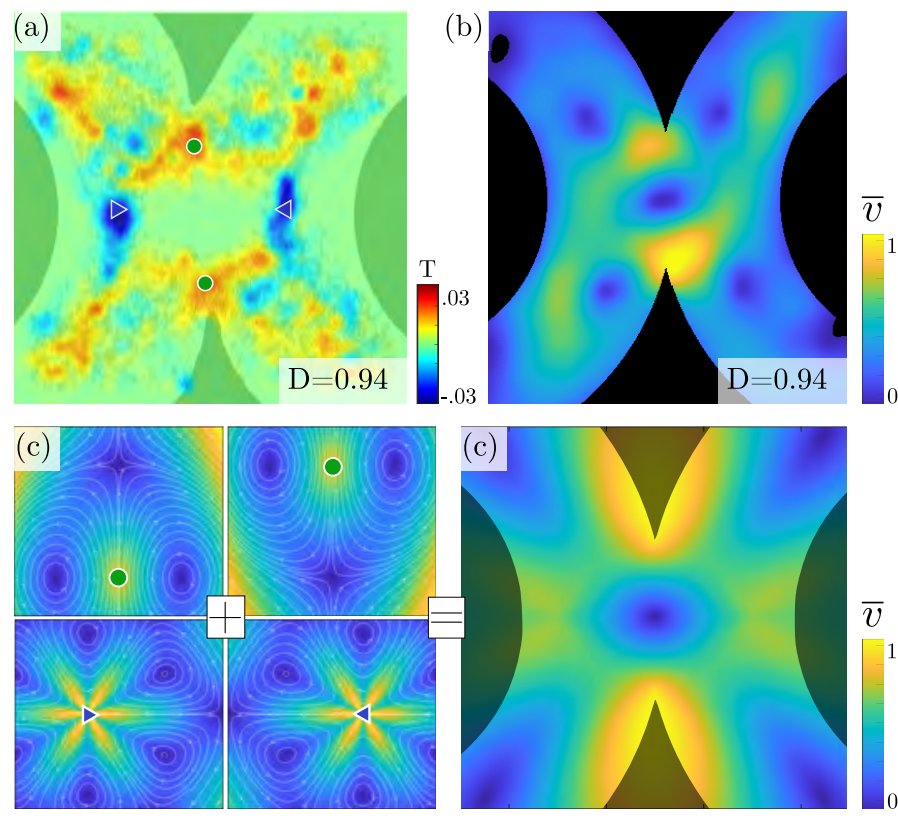

Fig. 5 Cross-talk between stagnation points in active flow and defect orientation. (a) Overlay of the local average topological charge $T$ at the center of two connected annuli with an inter-center distance $D / 2 R_{o}=0.94$. Regions with higher negative (resp. positive) topological charge are highlighted in blue (resp. red). Images are overlapped with a black mask around the region of interest, for a better visualization. (b) Corresponding velocity map. The speed has been normalized to 1 for simplicity. Dark blue spots are the signature of stagnation points, while bright yellow spots correspond to the regions with highest velocities. (c) Schematic reconstruction of the flow pattern. We superimpose the theoretical flow fields generated by two positive (top) and two negative (bottom) defects located at the positions defined in (a). See Methods section for the expression of the flow fields. (d) The resulting flow field both captures the stagnation points and the accelerated areas close to the positive defects. The mask overlay (black regions) does not correspond to any boundary condition imposed in the computation.

time as it enters in a CW motion around $t=100 \mathrm{~s}$. Interestingly, at the same time the flow order in annulus 2 dramatically drops to 0 . Therefore, at time $t=200 \mathrm{~s}$ annuli 1 and 3 are anti-correlated, and annulus 2 is turbulent. Eventually, around $t=300 \mathrm{~s}$, the annulus 2 enters a CCW rotation, while the flow order in annulus 3 drops to 0 . Such a process of alternating pair anti-correlations is not exclusive, and temporary events of transport in all annuli are sometimes observed (at times around $t=500 \mathrm{~s}$ in this particular experiment). Yet, the results suggest that the emerging flow pattern tends to favour two counter-rotating annuli facing a turbulent one. In other words, the system behaves as if frustration was accumulated in either of the three annuli, and exchanged over time. Such an intricate flow coupling requires further investigation. In particular, the time scale between the frustration exchanges could give information on what triggers the transitions. The corresponding dynamics is available in movie S5.

\section{Conclusions}

In this work, we have demonstrated that confining a twodimensional active nematic to a closed network of micro-channels allows us to transform the chaotic flows of the active material into 

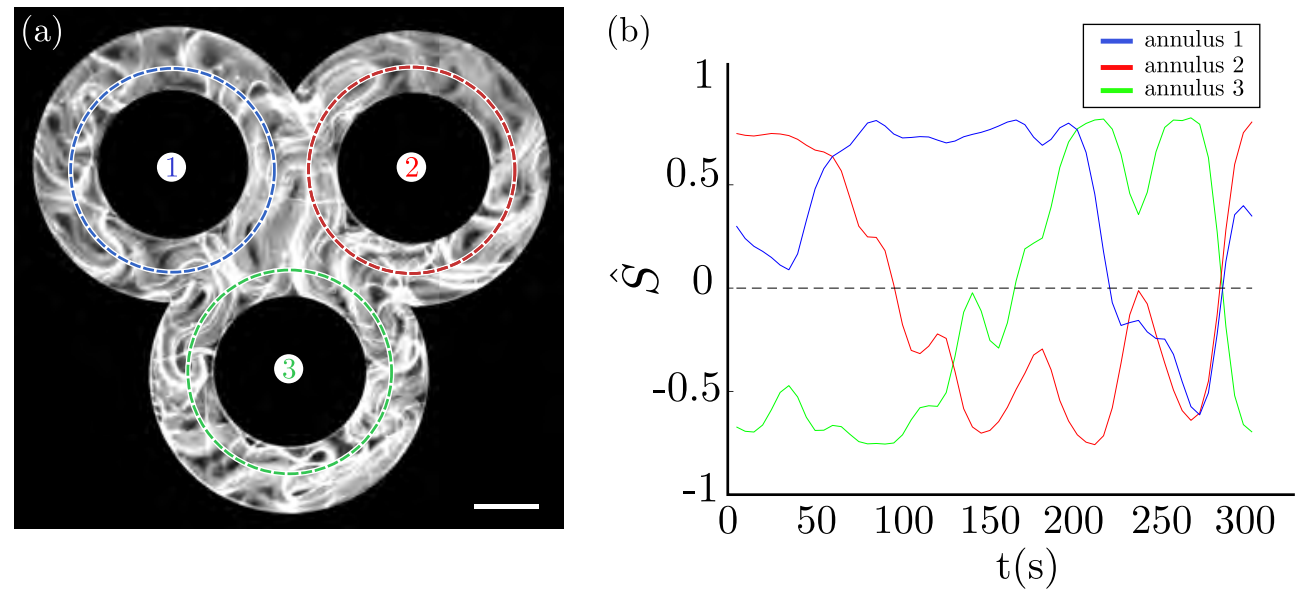

Fig. 6 Active flows in annuli connected in a genus three handlebody platform. (a) Three connected annuli of $80 \mu \mathrm{m}$ width with $D / 2 R_{o}=0.83$. The image is overlapped with a black mask around the region of interest, for a better visualization. Scale bar: $100 \mu \mathrm{m}$. (b) Flow order parameter as a function of time for each annulus in the assembled geometry.

controlled directional flows. The symmetry breaking observed in annuli, responsible for the transition between the chaotic and transport regimes, seems to stem from a complex coupling between the orientational (or defect) field and the flow field. On the one hand, the flow patterns spontaneously (and randomly) transit to directed transport. One the other hand, topological defects acquire a strong polar order, reminiscent of polar flocking. Currently, we cannot establish how both symmetry breaking events are related. One possibility is to invoke a polar flocking transition of the self-propelled positive defects, which would suggest that elastic interactions between defects favour a polar alignment that necessarily leads to directed flows. In this case, the symmetry breaking would originate away from the walls. Another possibility is a spontaneous hydrodynamic symmetry breaking unrelated to the nematic order, and which could transiently align the positive defect population. For example, a hydrodynamic source of symmetry breaking could be the emergence of edge currents, which are essential to understand bacterial vortices 30 64. Although these currents are driven by the thinnest layer of bacteria directly in contact with the walls, they completely determine the handedness of the bulk vortex. The importance of the walls in bulk active flows has also been raised in Ref ${ }^{39}$ and we have seen, in our experiments with active nematic layers, that the transport handedness can be controlled by small alterations of the wall properties (see Fig. S4).

The stabilisation of high charge topological defects in microfluidic devices has been reported for passive nematic liquid crystals, by generating hydrodynamic stagnation points of different topological charges at the center of star shaped microfluidic junctions 62 . In this work, we show that this idea can be extended to active systems, as demonstrated with $g=2$ and $g=3$ handlebody microchannels. A fundamental difference is that, here, the coupling between topological defects in the flow and orientational fields is spontaneously induced by the activity of the system and its interaction with the boundaries. More intricate flow patterns and dynamical states are expected to arise in larger microfluidic networks of connected annuli, as exemplified here for the case of $g=3$ handlebodies, where anti-synchronisation and frustration come to play. These results uncover the richness of active topological microfluidics, paving the way for the design of fully autonomous fluid circuits capable to perform complex tasks without external driving.

\section{Materials and Methods}

\subsection{Protein preparation}

Microtubules (MTs) were polymerized from heterodimeric $(\zeta, \beta)$ tubulin from bovine brain [a gift from Z. Dogic's group at Brandeis University (Waltham, MA)], incubated at $37^{\circ} \mathrm{C}$ for $30 \mathrm{~min}$ in aqueous M2B buffer ( $80 \mathrm{mM}$ Pipes, 1 mM EGTA, $2 \mathrm{mM} \mathrm{MgCl2}$ ) prepared with Milli-Q water. The mixture was supplemented with the reducing agent dithiothrethiol (DTT) (Sigma; 43815) and with guanosine-5-[( $\zeta, \beta)$-methyleno]triphosphate (GMPCPP) (Jena Biosciences; NU-405), a slowly hydrolysable analog of the biological nucleotide guanosine-5-triphosphate (GTP) that completely suppresses the dynamic instability of the polymerized tubulin. GMPCPP enhances spontaneous nucleation of MTs, obtaining high-density suspensions of short MTs $(1-2 \mu \mathrm{m})$. For fluorescence microscopy, 3\% of the tubulin was labeled with Alexa 647. Drosophila melanogaster heavy-chain kinesin-1 K401-BCCP6 His (truncated at residue 401, fused to biotin carboxyl carrier protein (BCCP), and labeled with six histidine tags) was expressed in Escherichia coli using the plasmid WC2 from the Gelles Laboratory (Brandeis University) and purified with a nickel column. After dialysis against $500 \mathrm{mM}$ imidazole aqueous buffer, kinesin concentration was estimated by means of absorption spectroscopy. The protein was stored in a $60 \%$ (wt/vol) aqueous sucrose solution at $-80^{\circ} \mathrm{C}$ for future use.

\subsection{Imaging}

Images were acquired using a Nikon spinning disk confocal microscope coupled to an Andor Zyla 4.2MP sCMOS camera. Typical videos were acquired with and exposure time of $500 \mathrm{~ms}$ at a frame rate of $2 \mathrm{fps}$. 

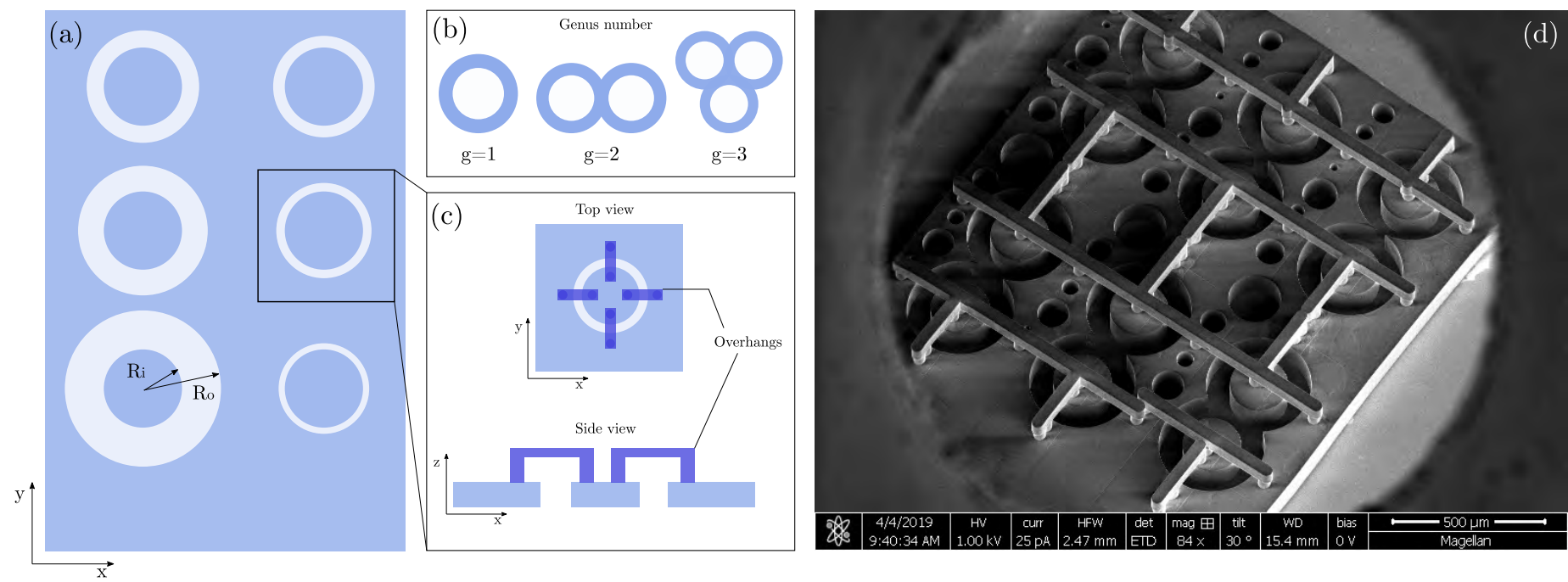

Fig. 7 Experimental Setup. (a) Sketch of a grid design in the case of annuli experiments. Six annuli with equal inner radius $R_{i}$ and varying outer radius $R_{o}$ are printed onto the same grid. In order to attach the inner disks to the grid, four overhangs per annulus are added on top of the grid. These overhangs have not been displayed here for more clarity. (b) Classification of assembled annuli in terms of the genus number. (c) Sketch of the detailed design of an annulus, showing the overhangs in top and side views. (c) Scanning Electron Microscope (SEM) image of a grid containing six Figures of 8. The picture is taken from the top with a small tilt allowing to clearly visualize the overhangs. In order to reduce the printing time and lighten the grid, additional disks have also been included around the figures of 8 . Scale bar: $500 \mu \mathrm{m}$.

\subsection{Grid Manufacturing}

The grids are printed using a two-photon polymerization printer, a Nanoscribe GT Photonic Professional device, with a negativetone photoresist IP-S (Nanoscribe GmbH, Germany) and a $25 \times$ objective. The grids were directly printed on silicon substrates without any preparation to avoid adhesion of the resist to the substrate (plasma cleaner of the substrate, for example, would increase the adhesion). After developping 30 minutes in Propylene Glycol Monomethyl Ether Acetate (PGMEA 99,5\%, Sigma Aldrich) and 5 minutes in isopropanol (Technical, VWR), a batch polymerization is performed with UV-exposure (5 min at $80 \%$ of light power). After printing onto a silicon wafer, the grids are bound to a vertical glass capillary with a UV-curable glue. The capillary is then delicately manipulated to detach the grids from the printing support. The grids are washed in three steps (isopropanol, DI water, ethanol) and dried with a nitrogen stream before each experiment. The thickness of the grids is $100 \mu \mathrm{m}$, to ensure good mechanical resistance. We have used grids with a variable number of connected annuli, leading to three type of micro-devices with different geometries and topologies: toroidal (single annulus), figure of 8 (two connected annuli) and trefoillike (three connected annuli) as shown in (Fig. 7(a, b)). Threedimensional printed overhangs ensure the mechanical integrity of the devices (Fig. 7(c, d)). In the case of single annuli, the inner radius $R_{i}$ was set to $150 \mu \mathrm{m}$. The outer radius was varied in order to explore a range of width between 40 and $200 \mu \mathrm{m}$. In the case of connected annuli, both $R_{i}$ and $R_{o}$ were set so that the width was kept at $80 \mu \mathrm{m}$. This choice is justified by the fact that such a width corresponds to the transition between directed transport and switching states, ensuring rich dynamics. Each grid contains multiple geometries, typically six at a time as shown in Fig. 7(d), in such a way that simultaneous experiments can be performed with the same active nematic preparation, thus ensuring that material parameters remain unchanged when comparing different confinement conditions. The micropatterning has a resolution of around $50 \mathrm{~nm}$, much higher than the needs of the experiment. The boundary conditions also appear to be well defined. We never observe microtubules sticking to the wall. As shown by the flow profiles measured in the shear state, the shear profile extends up to the wall, attesting for a free slip boundary condition. The grids are $100 \mu \mathrm{m}$ thick, while the thickness of the active nematic layer is of the order of a few microns. When the grid comes to the interface, we observe that the bound interface tends to lie somewhere between the top and bottom of the grid. When the grid impacts the interface, uncontrolled flows may destabilize the active nematic layer. These flows mostly depend on the parallelism between the micro-device and the fluid interface when they come into contact, but also on the wetting properties of the grid. The parallelism is relatively easy to control with micro-screw adjustment. On the other hand, the wetting properties of the grid are still under scrutiny. In our experience, the surface of the grids deteriorates after the first use, therefore the samples are considered single-use.

\subsection{Active Gel preparation}

Biotinylated kinesin motor protein and tetrameric streptavidin (Invitrogen; 43-4301) aqueous suspensions were incubated on ice for $30 \mathrm{~min}$ at the specific stoichiometric ratio $2: 1$ to obtain kinesin-streptavidin motor clusters. MTs were mixed with the motor clusters that acted as cross-linkers, and with ATP (Sigma; A2383) that drove the activity of the gel. The aqueous dispersion contained a nonadsorbing polymeric agent (PEG, 20 kDa; Sigma; 95172) that promoted the formation of filament bundles through depletion. To maintain a constant concentration of ATP during 
the experiments, an enzymatic ATP-regenerator system was used, consisting on phosphoenolpyruvate (PEP) (Sigma; P7127) that fueled pyruvate kinase/lactate dehydrogenase (PK/LDH) (Invitrogen; 434301) to convert ADP back into ATP. Several antioxidant components were also included in the solution to avoid protein denaturation, and to minimize photobleaching during characterization by means of fluorescence microscopy. Anti-oxydant solution 1 (AO1) contained $15 \mathrm{mg} / \mathrm{mL}$ glucose and 2.5M DTT. Antioxidant solution 2 contained $10 \mathrm{mg} / \mathrm{mL}$ glucose oxydase (Sigma G2133) and $1.75 \mathrm{mg} / \mathrm{mL}$ catalase (Sigma, C40). Trolox (Sigma, 238813) was used as an additional anti-oxidant. A high-salt concentration solution $\mathrm{M} 2 \mathrm{~B}$ was used to raise the $\mathrm{MgCl}_{2}$ concentration. The PEG-based triblock copolymer surfactant Pluronic F-127 (Sigma; P-2443) was added to procure a biocompatible water/oil interface in subsequent steps. Buffer for stock solutions of PEP, DTT, ATP, PEG and Streptavidin was M2B, and we added $20 \mathrm{mM}$ of $\mathrm{K}_{2} \mathrm{HPO}_{4}$ to the buffer of Catalase, Glucose, Glucose Oxydase and Trolox. A typical recipe is summarized in Table 1 .

\begin{tabular}{l|l|l} 
Compound & Stock solution & $\mathbf{v} / \mathbf{V}_{\text {total }}$ \\
\hline PEG & $12 \% \mathrm{w} / \mathrm{vol}$ & 0.139 \\
PEP & $200 \mathrm{mM}$ & 0.139 \\
High-salt M2B & $69 \mathrm{mM} \mathrm{MgCl}$ & 0.05 \\
Trolox & $20 \mathrm{mM}$ & 0.104 \\
ATP & $50 \mathrm{mM}$ & 0.03 \\
Catalase & $3.5 \mathrm{mg} \cdot \mathrm{ml}^{-1}$ & 0.012 \\
Glucose & $300 \mathrm{mg} \cdot \mathrm{ml}^{-1}$ & 0.012 \\
Glucose Oxydase & $20 \mathrm{mg} \cdot \mathrm{ml}^{-1}$ & 0.012 \\
PK/LDH & $600-1000$ units.ml & 0.03 \\
DTT & $0.5 \mathrm{M}$ & 0.012 \\
Streptavidin & $0.352 \mathrm{mg} \cdot \mathrm{ml}^{-1}$ & 0.023 \\
Kinesin & $0.07 \mathrm{mg} \cdot \mathrm{ml}^{-1}$ & 0.234 \\
Microtubules & $6 \mathrm{mg} \cdot \mathrm{ml}^{-1}$ & 0.167 \\
Pluronic & $17 \%$ & 0.027 \\
\hline
\end{tabular}

Table 1 Composition of all stock solutions, and their volume fraction in the final mixture.

\subsection{Defect detection}

Statistical information on defect position and defect orientation (Fig. 3 and Fig. S3 ) was obtained using the defect detection and tracking Matlab codes developed by Ellis et al. $\underline{40}$, coupled with a custom-made direction detection Matlab code derived from Vromans and Giomi's method 65. PIV measurements in Fig. S1 were performed using ImageJ PIV plugin 66 . The data were then processed using custom Matlab codes.

\subsection{Azimuthal polar order}

The averaged $\langle\cos \varphi(t)>$ in Fig. 3 is computed at a given time $t$ from all the positive defects detected in the annulus for a sliding window of 20 time steps. Statistics are obtained from a video of 600 frames at 2 frames/s. The average number of defects per frame was 18 . The average lifetime of a given defect track was 40 frames. As a consequence, the number of independent defects in the statistics $N_{i}$ is estimated around $N_{i} \sim 600 / 40 \times 18=270$ defects.
A more detailed statistical analysis of the distribution of defect orientations $\varphi$ is displayed in Fig. S3.

\subsection{Flow-order parameter $\hat{S}$}

For each frame, we select the pixels located at the center of the annulus (green line) in Fig. 2 (a). This pixel ring is then unwrapped to a 360-pixel line, where the intensity of pixel $\theta \in[0,360]$ is equal to the average intensity of the pixels located between the angles $\theta$ and $\theta+1$ around the ring. This process is repeated at each time frame, and the results are stacked on a time-series image as shown in Fig. 2 (b). As the active nematics moves, the fluorescence intensity patterns follow the positions of the microtubules. If the active nematics is in a transport state at time $t$, the intensity gradients in the time series image will exhibit stripes, whose slope (negative of positive) gives information on the handedness of the rotation (clockwise or anti-clockwise). The symmetry breaking is visualised by computing the FFT of the time series image at times $[t-\Delta t, t+\Delta t]$ with $\Delta t=20 \mathrm{~s}$. $\Delta t$ needs to be large enough to actually capture spatio-temporal dynamics, and small enough to get a suitable time resolution. A typical FFT image for clockwise transport is shown in Fig. 2 (c). If we notice that the FFT image is symmetric with respect to its center, we can now divide it in 4 panels, as shown in the inset of Fig. 2 (f). The top-left panel is symmetrical to the bottom-right one, and the bottom-left panel is symmetrical to the top-right one. As a consequence, all the information is available in the top-right and bottom-right panels. Looking at the FFT image for a clockwise transport in Fig. 2 (c) one notices that the intensity of the bottom-right panel, $I_{-}$is higher than the intensity in the top-right panel, $I_{+}$. We define $\hat{S}$ as the normalized intensity difference between the two panels: $\hat{S}=\frac{I_{+}^{2}-I_{-}^{2}}{I_{+}^{2}+I_{-}^{2}}$. Therefore, positive (resp. negative) values of $\hat{S}$ corresponds to clockwise CW (resp. counter-clockwise CCW) flows. The closer to $|\hat{S}|=1$, the stronger the symmetry breaking. Typically, $|\hat{S}|=0.5$ already corresponds to highly directional flows, while when $|\hat{S}|=0.2$ the flow is considered chaotic.

\subsection{Computed flow fields}

Positive and negative defects have been proven to generate flow fields of the following form 63 .

$$
\begin{aligned}
& v_{+, x}^{a}=\frac{\alpha}{12 \eta}\{[3(R+r)+r \cos 2 \phi] \hat{x} \\
& v_{+, y}^{a}=\frac{\alpha}{12 \eta}[r \sin 2 \phi] \hat{y} \\
& v_{-, x}^{a}=\frac{\alpha r}{12 \eta R}\left\{\left[\left(\frac{3}{4} R-r\right) \cos 2 \phi-\frac{R}{5} \cos 4 \phi\right] \hat{x}\right. \\
& v_{-, y}^{a}=-\frac{\alpha r}{12 \eta R}\left[\left(\frac{3}{4} R-r\right) \sin 2 \phi+\frac{R}{5} \sin 4 \phi\right] \hat{y} .
\end{aligned}
$$

We have computed these flow patterns in Matlab setting $\frac{\alpha}{12 \eta}=1$ and $R=0.8$. The half-width of the constructed images corresponds to $r=1$. We have then placed each source at a distance $d=0.4$ from the image center. The flow fields have been rotated in order to account for the observed orientation of the defects. 


\section{Acknowledgements}

We are indebted to the Brandeis University Materials Research Science and Engineering Centers (MRSEC) Biosynthesis facility for providing the tubulin. We thank M. Pons, A. LeRoux, G. Iruela, P. Guillamat and B. Martínez-Prat (University of Barcelona) for assistance in the expression of motor proteins. We also thank P. Ellis and A. Fernandez-Nieves for kindly sharing all their image processing and defect detection algorithms. J.H., J.I.-M and F.S. acknowledge funding from Ministerio de Economia, Industria y Competitividad, Spain (project FIS2016-78507-C2-1-P, Agencia Estatal de Investigación/European Regional Development Fund). J.H. acknowledges funding from the European Union's Horizon 2020 research and innovation program under grant agreement no. 674979-NANOTRANS. S. Brandeis University MRSEC Biosynthesis facility is supported by NSF MRSEC DMR-1420382. T.L-L. acknowledges funding from the French Agence Nationale de la Recherche (Ref. ANR-13-JS08-006-01). Gulliver and PMMH labs of ESPCI acknowledge funding from 2015 Grant SESAME MILAMIFAB (Ref. 15013105) for the acquisiton of a Nanoscribe GT Photonic Professional device.

\section{Conflict of interest}

The authors declare no conflict of interest.

\section{References}

1 S. Ramaswamy, Annual Review of Condensed Matter Physics, 2010, 1, 323-345.

2 M. C. Marchetti, J. F. Joanny, S. Ramaswamy, T. B. Liverpool, J. Prost, M. Rao and R. A. Simha, Reviews of Modern Physics, 2013, 85, 1143-1189.

3 M. O. Din, T. Danino, A. Prindle, M. Skalak, J. Selimkhanov, K. Allen, E. Julio, E. Atolia, L. S. Tsimring, S. N. Bhatia and J. Hasty, Nature, 2016, 536, 81-85.

4 R. Di Leonardo, L. Angelani, D. Dell'Arciprete, G. Ruocco, V. Lebba, S. Schippa, M. P. Conte, F. Mecarini, F. De Angelis and E. Di Fabrizio, Proceedings of the National Academy of Sciences, 2010, 107, 9541-9545.

5 A. Souslov, B. C. van Zuiden, D. Bartolo and V. Vitelli, Nature Physics, 2014, 13, 1091-1094.

6 E. Karzbrun, A. M. Tayar, V. Noireaux and R. Bar-Ziv, Science, 2014, 345, 829-832.

7 F. G. Woodhouse and J. Dunkel, Nature Communications, 2017, 8, 15169.

8 D. Needleman and Z. Dogic, Nat. Rev. Mat., 2017, 2, 17048.

9 J. Prost, F. Jülicher and J. F. Joanny, Nat. Phys., 2015, 11, 111-117.

10 A. Doostmohammadi, J. Ignés-Mullol, J. M. Yeomans and F. Sagués, Nat. Commun., 2018, 9, 3246.

11 T. Sanchez, D. T. Chen, S. J. DeCamp, M. Heymann and Z. Dogic, Nature, 2012, 491, 431-434.

12 G. Henkin, S. J. DeCamp, D. T. Chen, T. Sanchez and Z. Dogic, Philos. Trans. A: Math. Phys. Eng. Sci., 2014, 372, 0142.

13 S. J. DeCamp, G. S. Redner, A. Baskaran, M. F. Hagan and Z. Dogic, Nat. Matter., 2015, 14, 1110-1115.
14 P. Guillamat, J. Ignés-Mullol and F. Sagués, Proc. Natl. Acad. Sci., 2016, 113, 5498-502.

15 L. M. Lemma, S. J. Decamp, Z. You, G. L. and Z. Dogic, Soft Matter, 2019, 15, 3264-3272.

16 B. Martínez-Prat, J. Ignés-Mullol, J. Casademunt and F. Sagués, Nature Physics, 2019, 15, 362.

17 L. M. Pismen, Phys. Rev. E, 2013, 88, 050502.

18 L. Giomi, M. J. Bowick, X. Ma and M. C. Marchetti, Phys. Rev. Lett., 2013, 110, 228101.

19 S. P. Thampi, R. Golestanian and J. M. Yeomans, Phys. Rev. Lett., 2013, 111, 118101.

20 S. P. Thampi, R. Golestanian and J. M. Yeomans, Europhys. Lett., 2014, 105, 18001.

21 L. Giomi, Phys. Rev. X, 2015, 5, 031003.

22 E. J. Hemingway, P. Mishra, M. C. Marchetti and S. M. Fielding, Soft Matter, 2016, 12, 7943.

23 L. M. Pismen and F. Sagués, Euro. Phys. J. E, 2017, 40, 92.

24 S. Shankar, S. Ramaswamy, M. C. Marchetti and M. J. Bowick, Physical Review Letters, 2018, 121, 108002.

25 M. James, W. J. T. Bos and M. Wilczek, Physical Review E Statistical, Nonlinear, and Soft Matter Physics, 2018, 061101, 061101.

26 D. J. G. Pearce, Physical Review Letters, 2019, 122, 227801.

27 F. G. Woodhouse and R. E. Goldstein, Physical Review Letters, 2012, 109, 168105.

28 H. Wioland, F. G. Woodhouse, J. Dunkel, J. O. Kessler and R. E. Goldstein, Phys. Rev. Lett., 2013, 110, 268102.

29 E. Lushi, H. Wioland and R. E. Goldstein, PNAS, 2014, 111, 9733.

30 H. Wioland, F. G. Woodhouse, J. Dunkel and R. E. Goldstein, Nat. Phys., 2016, 12, 341-345.

31 H. Wioland, E. Lushi and R. E. Goldstein, New Journal of Physics, 2016, 18, 075002.

32 F. J. Segerer, F. Thüroff, A. Piera Alberola, E. Frey and J. O. Rädler, Physical Review Letters, 2015, 114, 228102.

33 G. Duclos, C. Erlenkämper, J. F. Joanny and P. Silberzan, Nature Physics, 2017, 13, 58.

34 W. Xi, S. Sonam, T. Beng Saw, B. Ladoux and C. Teck Lim, Nature Communications, 2017, 8, 1517.

35 G. Duclos, C. Blanch-Mercader, V. Yashunsky, G. Salbreux, J. F. Joanny, J. Prost and P. Silberzan, Nature Physics, 2018, 14, 728.

36 K. Suzuki, M. Miyazaki, J. Takagi, T. Itabashi and S. Ishiwata, PNAS, 2017, 114, 2922-2927.

37 F. C. Keber, E. Loiseau, T. Sanchez, S. J. DeCamp, L. Giomi, M. J. Bowick, M. C. Marchetti, Z. Dogic and A. R. Bausch, Science, 2014, 345, 1135-9.

38 P. Guillamat, J. Ignés-Mullol and F. Sagués, Nat. Commun., 2017, 8, year.

39 K. T. Wu, J. B. Hishamunda, D. T. N. Chen, S. J. DeCamp, Y. W. Chang, A. Fernández-Nieves, S. Fraden and Z. Dogic, Science, 2017, 355, year.

40 P. W. Ellis, D. J. G. Pearce, Y.-W. Chang, G. Goldsztein, 
L. Giomi and A. Fernández-Nieves, Nat. Phys., 2018, 14, 85.

41 P. Guillamat, Ž. Kos, J. Hardoüin, J. Ignés-Mullol, M. Ravnik and F. Sagués, Sci. Adv., 2018, 4, eaao1470.

42 D. J. G. Pearce, P. W. Ellis, A. Fernandez-Nieves and L. Giomi, Physical Review Letters, 2019, 122, 168002.

43 A. Opathalage, M. M. Norton, M. P. N. Juniper, B. Langeslay, S. A. Aghvami, S. Fraden and Z. Dogic, Proceedings of the National Academy of Sciences, 2019, 116, 4788-4797.

44 J. Hardoüin, R. Hughes, A. Doostmohammadi, J. Laurent, T. Lopez-Leon, J. M. Yeomans, J. Ignés-Mullol and F. Sagués, Commun. Phys., 2019, 2, 121.

45 E. Tjhung, D. Marenduzzo and M. E. Cates, PNAS, 2012, 109, 12381-12386.

46 M. Ravnik and J. M. Yeomans, Phys. Rev. Lett., 2013, 110, 026001.

47 C. A. Whitfield, D. Marenduzzo, R. Voituriez and R. J. Hawkins, Euro. Phys. J. E, 2014, 37, 8.

48 L. Giomi and A. DeSimone, Phys. Rev. Lett., 2014, 112, 147802.

49 R. Sknepnek and S. Henkes, Physical Review E - Statistical, Nonlinear, and Soft Matter Physics, 2015, 91, 022306.

50 R. Zhang, Y. Zhou, M. Rahimi and J. J. De Pablo, Nature Communications, 2016, 7, 13483.

51 D. Khoromskaia and G. P. Alexander, New Journal of Physics, 2017, 19, 103043.

52 T. Gao and Z. Li, Phys. Rev. Lett., 2017, 119, 108002.

53 M. Leoni, O. V. Manyuhina, M. J. Bowick and M. C. Marchetti, Soft Matter, 2017, 13, 1257-1266.

54 M. Theillard, R. Alonso-Matilla and D. Saintillan, Soft Matter, 2017, 13, 363.

55 T. N. Shendruk, A. Doostmohammadi, K. Thijssen and J. M. Yeomans, Soft Matter, 2017, 13, 3853.

56 R. Green, J. Toner and V. Vitelli, Physical Review Fluids, 2017, 2, 104201.

57 M. M. Norton, A. Baskaran, A. Opathalage, B. Langeslay, S. Fraden, A. Baskaran and M. F. Hagan, Physical Review E, 2018, 97, 012702.

58 S. Chen, P. Gao and T. Gao, Journal of Fluid Mechanics, 2018, 835, 393-405.

59 A. Sengupta, Liquid Crystals Today, 2017, 24, 70-80.

60 P. Guillamat, J. Ignés-Mullol, S. Shankar, M. C. Marchetti and F. Sagués, Phys. Rev. E, 2016, 94, 060602.

61 P. Guillamat, J. Hardouin, B. M. Prat, J. Ignes and F. Sagues, J Phys Condens Matter, 2017.

62 L. Giomi, M. Ravnik and A. Sengupta, Proceedings of the National Academy of Sciences, 2017, 114, ES771.

63 L. Giomi, M. J. Bowick, P. Mishra, R. Sknepnek and M. C. Marchetti, Philosophical Transactions of the Royal Society A, 2014, 372, 20130365.

64 D. Nishiguchi, I. S. Aranson, A. Snezhko and A. Sokolov, Nat Commun, 2018, 9, 4486.

65 A. J. Vromans and L. Giomi, Soft matter, 2016, 12, 64906495.
66 Q. Tseng, E. Duchemin-Pelletier, A. Deshiere, M. Balland, H. Guillou, O. Filhol and M. Théry, Proceedings of the National Academy of Sciences, 2012, 109, 1506-1511. 